\title{
Biophysical Mechanisms That Govern the Vascularization of Microfluidic Scaffolds
}

Keith H. K. Wong, James G. Truslow, Aimal H. Khankhel, and Joe Tien

\section{CONTENTS}

6.1 Introduction 109

6.2 Formation, Vascularization, and Perfusion of Microfluidic Scaffolds ...................... 110

6.3 Biophysical Considerations of Scaffold Material Properties .................................... 112

6.4 How Physical Forces May Determine Vascular Stability........................................... 114

6.4.1 Prediction: An Increase in Transmural Pressure Stabilizes Vascular

Adhesion to the Scaffold ................................................................................. 114

6.4.2 Prediction: Drainage Stabilizes Vascular Adhesion, Especially When the Scaffold Is Hydraulically Resistive .......................................................... 117

6.4.3 Prediction: Strong Endothelial Barrier Function Promotes Vascular Stability ......................................................................................... 118

6.4.4 Prediction: Scaffold Stiffening Promotes Vascular Stability Independently of Inhibition of Gel Contraction.

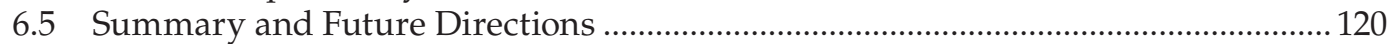

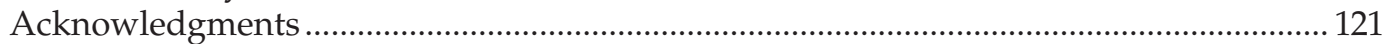

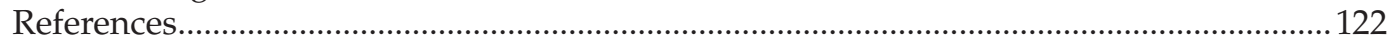

\subsection{Introduction}

Methods to engineer vascularized tissues have historically focused on chemical and/or biological strategies (Lovett et al. 2009). For instance, loading of scaffolds with vascular growth factors, functionalization of scaffolds with bioactive peptides, or incorporation of extracellular matrix (ECM) components into scaffolds can induce angiogenesis and vasculogenesis, the natural processes of vascular formation and growth that occur during development and wound healing [reviewed in (Bouhadir and Mooney 2001, Vailhé et al. 2001, Kaully et al. 2009)]. Likewise, cultures of differentiated vascular cells, mesenchymal stem cells, and/or blood-borne progenitor cells can reorganize to form long-lived vascular networks (Asahara et al. 1997, Koike et al. 2004, Au et al. 2008).

In comparison, physical approaches to vascularization (e.g., those that involve stress, stiffness, flow, and geometry) are much less well studied. The rationale for investigating such physical strategies lies in the possibility of obtaining complementary methods to tailor the formation and functionality of vascular networks. Recent work in vascular and nonvascular systems has indicated the importance of the physical microenvironment in 
biological function (Chen et al. 2004, Discher et al. 2005). While this work in mechanobiology has mostly focused on two-dimensional cultures, the underlying ideas also apply to three-dimensional systems (Alcaraz et al. 2004, Gjorevski and Nelson 2010). Studying how physical signals may affect or underlie vascularization of scaffolds will likely lead to a better understanding of how to manipulate the vascularization process for a desired outcome.

To this end, we and others have advocated forming microvessels in microfluidic scaffolds (those that contain open channels or networks), both as well-defined model systems for studying the role of physical signals on vascular function and as perfusable materials for potential implantation (Price and Tien 2009, Miller et al. 2012, Tien et al. 2013, Zheng et al. 2012). The high-resolution techniques that are used to create microfluidic scaffolds enable intricate control over the channel geometries and the perfusion rates (Wong et al. 2012); such control allows one to alter physical signals within a vascularized microfluidic scaffold precisely. Practically, a major advantage of microfluidic scaffolds is their capability to sustain immediate perfusion by virtue of the preexisting fluidic channels. In principle, this perfusion is required to maintain the viability of densely cellularized tissue constructs (Choi et al. 2007). This property of microfluidic scaffolds lies in sharp contrast to chemically- or biologically driven angiogenesis or vasculogenesis, both of which require at least several days to develop structures capable of sustaining perfusion (Shepherd et al. 2004, Tremblay et al. 2005, Chiu et al. 2012, Yeon et al. 2012).

This chapter summarizes our recent experimental studies of vascularization of microfluidic scaffolds, proposes a unified biomechanical theory of vascular stability in these scaffolds, and suggests some testable predictions for future investigation. It updates and elaborates upon the physical concepts introduced in a recent review (Tien et al. 2013).

\subsection{Formation, Vascularization, and Perfusion of Microfluidic Scaffolds}

Our experimental approach is to form microfluidic channels or networks inside hydrogels, and to culture endothelial cells within these channels under perfusion. The cultured cells will then grow to form confluent tubes with a circular cross section (Price and Tien 2009, 2011). We have chosen to use ECM hydrogels because they promote cell adhesion, in contrast to synthetic polymers that often require coupling with adhesive peptides before use.

The specific methods for forming the microfluidic structures vary with the channel geometry. For single channels, we typically rely on cylindrical needles as removable templates (Chrobak et al. 2006); for interconnected networks, we use micropatterned hydrogels as sacrificial materials that can be partially encapsulated and dissolved (Golden and Tien 2007) or as separate layers that can be bonded (Price et al. 2008). Once the channels have formed, their vascularization is straightforward albeit technically demanding. Below, we restrict the discussion to the vascularization of single channels (Chrobak et al. 2006) with and without accompanying drainage channels (Wong et al. 2013) because these simple geometries have allowed routine mathematical analyses of the fluid and solid mechanics of the vessels and scaffolds. For step-by-step experimental procedures, we refer the reader to published protocols (Price and Tien 2009, 2011).

To create single microfluidic channels, we form type I collagen or fibrin gels around a stainless-steel acupuncture needle (120- $\mu \mathrm{m}$-diameter, Japanese gauge 02), and remove 
(a)
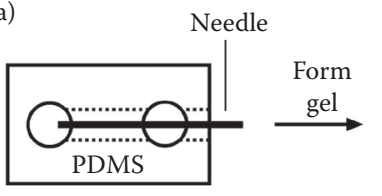

(b)

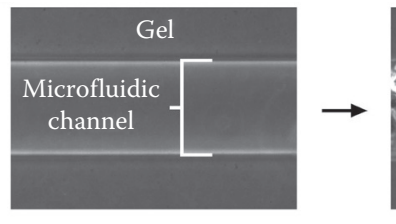

(c)

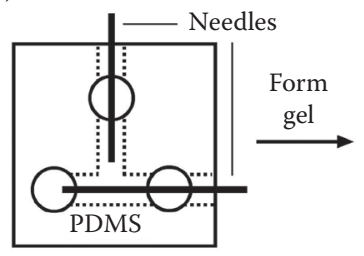

(d)

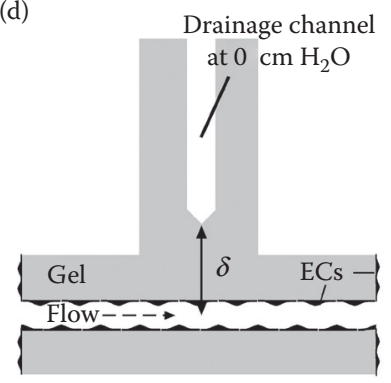

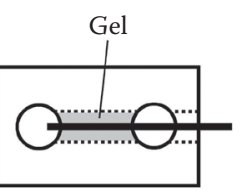

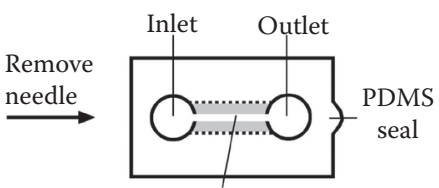

Microfluidic channel to be vascularized
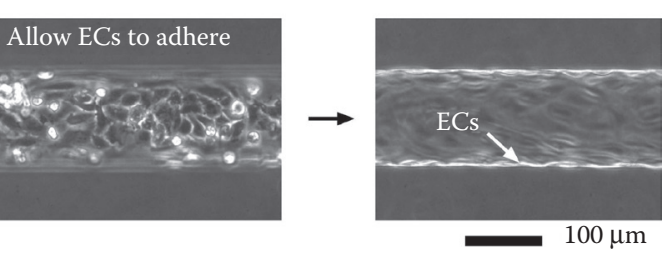

$100 \mu \mathrm{m}$

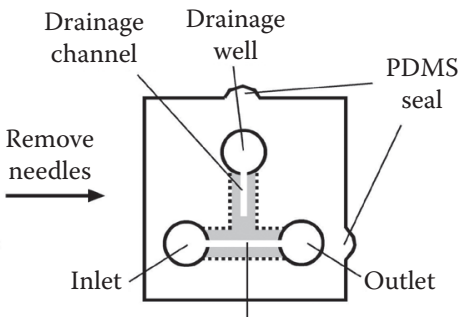

Microfluidic channel to be vascularized

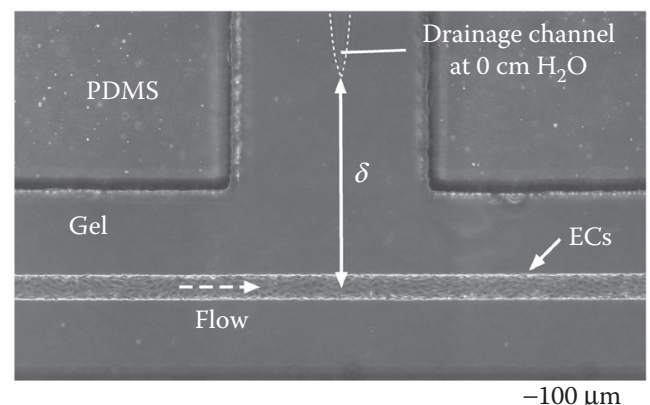

\section{FIGURE 6.1}

Strategies for forming endothelialized microfluidic scaffolds. (a) Fabrication of ECM scaffolds that contain a single microfluidic channel. The ECM hydrogel is formed around a stainless-steel needle (typically $120 \mu \mathrm{m}$ in diameter), which is then removed to yield a microchannel that interfaces with the inlet and outlet of the PDMS chamber. The channel is then seeded with endothelial cells. (b) Endothelial cells are cultured to confluence in microchannels under constant media perfusion. (c) Fabrication of scaffolds that contain an additional drainage channel. The drainage channel is formed perpendicularly to subject the gel to atmospheric pressure. (d) Phasecontrast image of a microvessel with a drainage channel in a fibrin gel. (Adapted with permission from Wong, K. H. K. et al. 2013. J Biomed Mater Res A, 101, 2181-90.)

the needle after gel polymerization to leave an open channel within the gel (Figure 6.1a). The gel is directly polymerized inside a polydimethylsiloxane (PDMS) chamber, which acts as a mechanical support and allows fluidic connections across the inlet and outlet of the vessel (Figure 6.1a). We vascularize these channels by introducing a suspension of blood microvascular endothelial cells, allowing them to adhere, connecting the inlet and 
outlet to media reservoirs at different pressures, and culturing the seeded channels under constant perfusion. We maintain steady flow by recycling effluent media once or twice daily to the reservoir that connects to the inlet. In general, a confluent endothelial tube (Figure 6.1b) forms within 1 day. The application of pressure differences of $1-12 \mathrm{~cm} \mathrm{H}_{2} \mathrm{O}$ across 7- to 9-mm-long vessels generates flow rates and wall shear stresses of $0.1-3 \mathrm{~mL} / \mathrm{h}$ and 1-30 dyn $/ \mathrm{cm}^{2}$, respectively (Chrobak et al. 2006, Price et al. 2010, Wong et al. 2010, Leung et al. 2012). This method precisely controls the lumenal (i.e., intravascular) pressure and its axial gradient, and can reliably maintain steady flow for weeks, but only if the endothelium remains adherent to the scaffold.

Recently, we have developed methods to control the fluid pressure of the scaffold outside the vessel (i.e., the extravascular or interstitial fluid pressure) (Wong et al. 2013). This work was inspired by the design and function of the lymphatic system in vivo, in which lowpressure endothelial tubes drain excess fluid from the tissue space and thus maintain the interstitial pressure at near-atmospheric pressures (Schmid-Schönbein 1990). By incorporating empty (i.e., nonvascularized) microfluidic channels that were independently held at atmospheric pressure (Figure 6.1c and d), we could independently modulate the intra- and extravascular pressures and thereby study how the transmural pressure (i.e., the difference between lumenal and interstitial pressures) affected the stability of engineered blood microvessels. Although lymphatics in vivo are lined by specialized (lymphatic) endothelium, the empty channels appear to serve as an effective replacement to provide drainage function in microfluidic scaffolds.

\subsection{Biophysical Considerations of Scaffold Material Properties}

Investigations of the vascularization of microfluidic hydrogels that are prepared under different conditions and with different structures have indicated that the physical properties of the scaffolds play an important role in determining the effectiveness of vascularization (Chrobak et al. 2006). The two principal relevant properties are the scaffold stiffness and pore size.

Depending on the subsequent degree of handling, microfluidic scaffolds require various degrees of mechanical stiffness. In our design, which encases the gel inside a PDMS chamber and requires no direct gel manipulation after polymerization, a low-density collagen gel with a shear modulus around hundreds of pascals is sufficient (Chrobak et al. 2006). If the fabrication process involves manual transfer of hydrogels, then a greater mechanical strength may be preferred to withstand deformation; for such cases, gel densities much larger than $10 \mathrm{mg} / \mathrm{mL}$ with moduli values on the order of $10-50 \mathrm{kPa}$ can be advantageous (Ling et al. 2007, Nichol et al. 2010).

The physical properties of the scaffold also control the degree of cell-mediated remodeling after seeding (Figure 6.2a). We have found that collagen polymerization conditions that form soft gels $\left(3 \mathrm{mg} / \mathrm{mL}\right.$, gelled at $\left.37^{\circ} \mathrm{C}\right)$ result in irreversible vessel contraction. Depending on the softness of the gel, the degree of contraction may be so severe that perfusion desists under practically attainable pressures. The contraction appears to result from the inherent contractility of the endothelium, which can be substantial in cultured cells (Kolodney and Wysolmerski 1992). If one models the endothelium as a contractile thin film, then the inward radial stress that results from cell contraction is given by the law of Laplace as $\gamma_{E C} / r$, where $\gamma_{E C}$ is the endothelial contractility and $r$ is the 
(a)
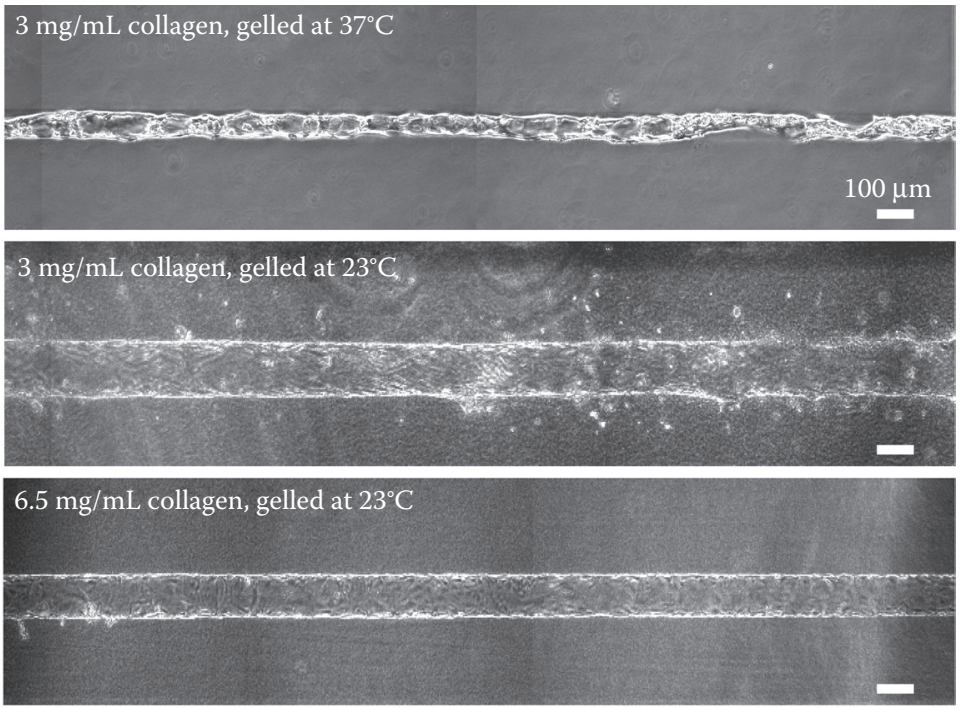

(b)
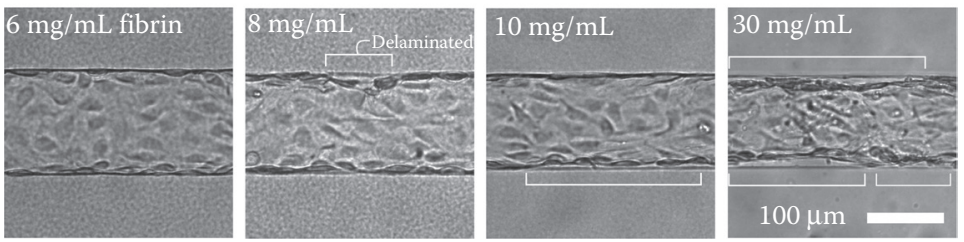

FIGURE 6.2

Effects of scaffold material properties on vessel formation. (a) In $3 \mathrm{mg} / \mathrm{mL}$ collagen gels that are polymerized at $37^{\circ} \mathrm{C}$, microvessels contract by day 2 postseeding. In gels that are polymerized at $23^{\circ} \mathrm{C}$, microvessels do not contract, but endothelial cells invade the surrounding collagen matrix. Invasion of endothelial cells is significantly less when collagen density is increased to $6.5 \mathrm{mg} / \mathrm{mL}$. (b) Microvessels that are formed in fibrin gels of increasing densities (from 6 to $30 \mathrm{mg} / \mathrm{mL}$ ) show a higher tendency to delaminate (i.e., detach) from the scaffold. (Adapted with permission from Chrobak, K. M., Potter, D. R., and Tien, J. 2006. Microvasc Res, 71, 185-96; Wong, K. H. K. et al. 2013. J Biomed Mater Res A, 101, 2181-90.)

contracted vascular radius. This stress leads to an inward fractional change in vessel radius of approximately $\gamma_{E C} / 2 G r$, where $G$ is the shear modulus of the scaffold (Barber 2010). That is

$$
1-\frac{r}{r_{0}}=\frac{\gamma_{E C}}{2 G r}
$$

where $r_{0}$ is the initial (not contracted) vascular radius. This equation has no solution when

$$
G<\frac{2 \gamma_{E C}}{r_{0}}
$$

Under this condition, the vessel will contract catastrophically. For the vessels and scaffolds that we typically use, $2 \gamma_{E C} / r_{0}$ is on the order of $100 \mathrm{~Pa}$, and scaffolds that are softer than this threshold cannot withstand endothelial contraction. We note that smaller vessels will require proportionally stiffer scaffolds to avoid this effect. 
Conditions that form porous gels $\left(3 \mathrm{mg} / \mathrm{mL}\right.$, gelled at $\left.23^{\circ} \mathrm{C}\right)$ lead to migration of single cells into the surrounding gel (Chrobak et al. 2006); this invasion can be inhibited by increasing gel density to decrease the pore size (Figure 6.2a). Empirically optimized conditions for microfluidic collagen scaffolds are gelation at room temperature $\left(\leq 25^{\circ} \mathrm{C}\right)$ and the use of gel concentrations that are $\geq 6.5 \mathrm{mg} / \mathrm{mL}$, which lead to shear moduli of $\sim 400 \mathrm{~Pa}$ and hydraulic conductivity ( $K$; a measure of pore size) of $\sim 3 \times 10^{-8} \mathrm{~cm}^{4} /$ dyn $\cdot \mathrm{s}$ (Wong et al. 2010). Although one can further stiffen scaffolds and reduce the pore size by increasing the scaffold concentration, such changes may not be desirable; in particular, we have recently found that a very low hydraulic conductivity in the scaffold can inhibit the transport of interstitial fluid and thereby destabilize vessels (see below).

\subsection{How Physical Forces May Determine Vascular Stability}

When scaffolds are stiff enough to resist contraction and dense enough to resist invasion, vessels that are formed within them will remain open, and perfusion will be stable, for 2-3 days. In the absence of additional stabilizing signals, however, long-term perfusion is not guaranteed. The endothelium can gradually delaminate from the microfluidic channel over the course of a week (Figure 6.2b); flow rates will likewise decrease, and often substantially. To understand the origin of this effect, and to discover signals that can promote vascular stability, we have manipulated the hydrodynamic stresses across the vessel wall directly (through variation of lumenal pressure $P_{\text {lumen }}$ and interstitial pressure $P_{\text {scaffold }}$ ) or indirectly (through changes in transvascular fluid flow via alterations in endothelial permeability) (Price et al. 2010, Leung et al. 2012, Wong et al. 2010, 2013).

These experiments have pointed to a physical basis of vascular stability. In engineered microvessels, the relevant stresses involved in vascular stability include the transmural pressure $P_{T M} \equiv P_{\text {lumen }}-P_{\text {scaffold }}$, stress due to endothelial contraction $\gamma_{E C} / r$, and endothelialscaffold adhesion $\sigma_{a d h}$ (Figure 6.3a). The simplest model postulates that a phenomenological force balance determines vascular stability:

$$
\left(P_{\text {lumen }}-P_{\text {scaffold }}\right)+\sigma_{\text {adh }}=P_{T M}+\sigma_{a d h}>\frac{\gamma_{E C}}{r}
$$

When this inequality is satisfied, the stabilizing stresses due to transmural pressure and scaffold adhesion outweigh the destabilizing stress due to contractility, and the vessel will remain adherent to the scaffold (Tien et al. 2013). In the following section, we describe experimental results in the context of this mechanical model, and provide evidence that transmural pressure is one of the predominant predictors of vascular stability. In microvascular networks in vivo, transmural pressure is an important driving force in transvascular filtration of fluid (Michel and Curry 1999), and it dictates microvascular perfusion by controlling the diameter and hence the fluidic resistance of capillaries (Lee and Schmid-Schönbein 1995).

\subsubsection{Prediction: An Increase in Transmural Pressure Stabilizes Vascular Adhesion to the Scaffold}

The mechanical model of vascular stability predicts that transmural pressure $P_{T M}$ acts to stabilize the vessel lumen, since an increase in $P_{T M}$ makes the inequality in Equation 6.3 more 
(a) Mechanical analysis

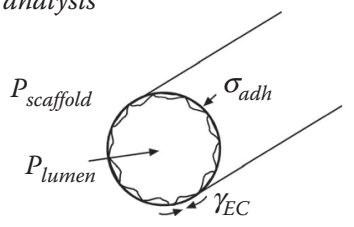

$\begin{gathered}\text { Condition } \\ \text { for stability: }\end{gathered} \quad P_{\text {lumen }}-P_{\text {scaffold }}>\frac{\gamma_{E C}}{r}-\sigma_{\text {adh }}$

(b) Energy analysis

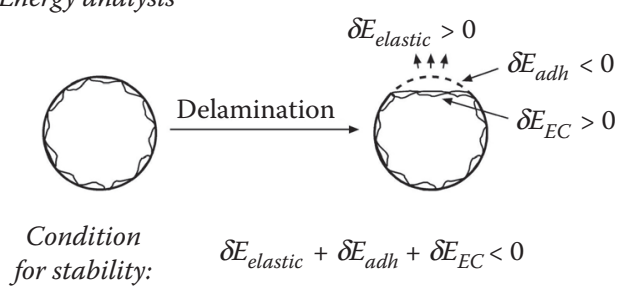

FIGURE 6.3

Proposed models of vessel stability. (a) Mechanical force balance: Vessels are stable when the total stabilizing stresses-transmural pressure (i.e., lumenal pressure $P_{\text {lumen }}$ minus scaffold pressure $P_{\text {scaffold }}$ ) and cell adhesion stress $\sigma_{a d h}$-are greater than the destabilizing contractile stress $\gamma_{E C} / r$. (b) Energy analysis: Vessels are stable when the energy required to break cell-scaffold adhesion bonds $\left|\delta E_{a d h}\right|$ is greater than the total energy released from elastic recoil of the scaffold $\delta E_{\text {elastic }}$ and retraction of endothelium $\delta E_{E C}$.

likely to be satisfied. In a deformable scaffold, the transmural pressure will cause the vessel to distend if $P_{T M}-\gamma_{E C} / r$ is positive. Thus, one can estimate $P_{T M}$ by measuring the degree of radial distension of the vessel and the elastic modulus of the scaffold. Consistent with the prediction, tubes that distend outward are invariably stable over the long term (Price et al. 2010).

In contrast, we found in early experiments that endothelial tubes that were perfused under nearly zero-flow rates invariably delaminated from the scaffold over time. In this configuration, all pressures are held small so that the flow rate is very low, and the transmural pressure is close to zero. This pair of observations-stability when tubes distend, instability when they do not-is consistent with the theory that a positive transmural pressure is required for vascular stability. Moreover, since endothelial delamination occurs when $P_{T M}$ is nearly zero, the endothelial contractile stress $\left(\gamma_{E C} / r\right)$ is likely to be greater than the cell adhesion stress $\left(\sigma_{a d h}\right)$. Direct measurement of endothelial contractility under nearly zero-flow conditions indicates that $\gamma_{E C} / r$ is equivalent to an inward radial strain of $\sim 5 \%$ and a tensile radial stress of $\sim 40 \mathrm{~Pa}$ in $\sim 8 \mathrm{mg} / \mathrm{mL}$ collagen gels (Wong et al. 2010).

To verify that a positive transmural pressure is required to prevent endothelial delamination under normal flow conditions, we designed experiments to artificially hold $P_{\text {scaffold }}$ nearly equal to $P_{\text {lumen }}$, so that $P_{T M}$ is nearly zero everywhere along the vessel. This pressure condition was achieved by placing an empty channel near and parallel to the vessel, and by subjecting both this channel and the vessel to the same perfusion pressures (Figure 6.4a). With this setup, the same axial pressure distributions will exist within the vessel lumen and the scaffold, thereby greatly reducing the transmural pressure. We found that endothelial tubes that are located adjacent to an empty channel held at a similar pressure grow to confluence, but invariably delaminate by day 3 postseeding (Price et al. 
(a)

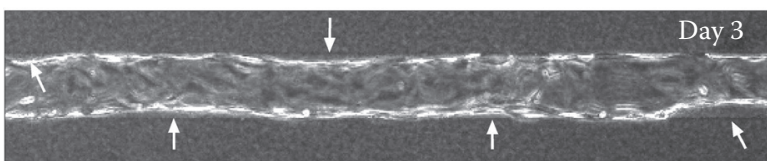

Delamination

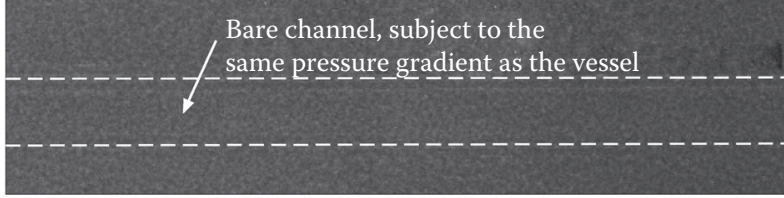

$-100 \mu \mathrm{m}$

(b)

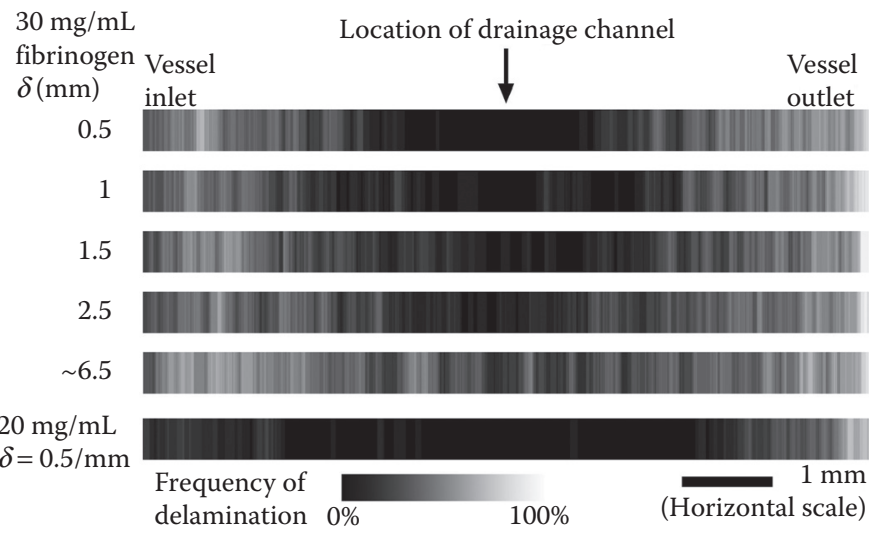

(c) Predicted $P_{T M}\left(\mathrm{~cm} \mathrm{H}_{2} \mathrm{O}\right)$

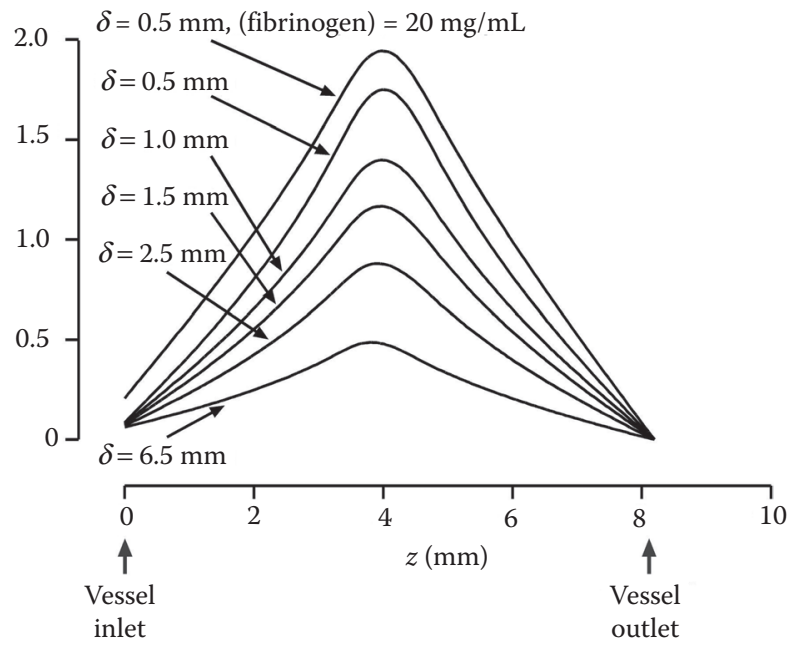

FIGURE 6.4 
2010). Mechanically, this situation is similar to that of compartment syndrome, in which perfusion is impaired within an enclosed tissue (e.g., muscle surrounded by fascia) when the tissue pressure is elevated.

\subsubsection{Prediction: Drainage Stabilizes Vascular Adhesion, Especially When the Scaffold Is Hydraulically Resistive}

A second prediction of the mechanical model is that the hydraulic resistance of the scaffold controls the level of vascular stability. This resistance lies in series with the resistance across the vessel wall; thus, in scaffolds of higher resistance, the pressure gradients in the scaffold are higher, the pressure differences across the vessel wall (i.e., $P_{T M}$ ) are lower, and the vessel is less stable (Truslow et al. 2009). Indeed, we have experimentally found in fibrin gels of various densities that vascular stability is anticorrelated with scaffold permeability $K$ (Figure 6.2b) (Wong et al. 2013). Vessels in fibrin gels with $K$ above $\sim 10^{-9} \mathrm{~cm}^{4}$ / dyn $\cdot s$ are stable, but not in gels with lower permeabilities.

In resistive scaffolds, the introduction of empty channels to directly lower the pressure within the scaffold should increase $P_{T M}$ and hence enhance vascular stability. These artificial lymphatic-like structures serve to drain fluid from the scaffold at a small, but measurable, rate (Wong et al. 2013). By placing such drainage channels perpendicular to the vessel axis, we have shown that drainage effectively stabilizes the portions of the vessel that lie sufficiently close to the drainage channel (within $\sim 1 \mathrm{~mm}$, for a drainto-vessel distance of $\sim 0.5 \mathrm{~mm}$ in $30 \mathrm{mg} / \mathrm{mL}$ fibrin gels) (Figure 6.4b) (Wong et al. 2013). Placing the drainage channel further from the vessel wall led to a gradual loss of stabilization. Moreover, when the drain-to-vessel distance was held constant while the scaffold permeability was varied, the stabilizing effect of drainage was stronger in scaffolds of greater porosity $(20 \mathrm{mg} / \mathrm{mL}$ vs. $30 \mathrm{mg} / \mathrm{mL}$ fibrin; Figure $6.4 \mathrm{~b})$. These results are all consistent with numerical models of the pressure distribution across the endothelium and in the scaffold (Figure 6.4c), and suggest that a large transmural pressure can be obtained by minimizing the hydraulic resistance from the vessel wall to low-pressure drainage (Truslow et al. 2009).

By comparing the experimentally determined delamination probabilities along the vessel axes with the computationally calculated transmural pressure distributions, we have obtained the first estimate of the minimum transmural pressure $\gamma_{E C} / r-s_{a d h}$ that is required for vascular stability (Wong et al. 2013). Given the uncertainties in the hydraulic properties of the vessel wall, we estimated the minimum required transmural pressure to be $40-140 \mathrm{~Pa}$ in fibrin gels. This range is remarkably consistent with the $\sim 40 \mathrm{~Pa}$ estimate of endothelial contractile stress, which strengthens our claim that the delicate

FIGURE 6.4

Increases in transmural pressure promote vessel stability. (a) Forcing the transmural pressure to be nearly zero along the entire vessel (by subjecting the gel to the same axial pressure distribution as the vessel) leads to endothelial delamination. (b) In contrast, maintenance of a positive transmural pressure by draining the hydrogel of interstitial fluid (refer to Figure 6.1d for experimental setup) stabilizes vessels. The presence of a drainage channel (held at atmospheric pressure) in the middle of the scaffold prevents delamination in the vicinity of the channel. As the drainage channel is placed further away from the vessels (i.e., as $\delta$ increased), the stabilized region gradually shortens and eventually disappears. (c) Numerical modeling predicts that transmural pressure is highest near the middle of the vessel, consistent with experimental results. Transmural pressure decreases sharply as the drainage channel is moved away from the vessel wall. Fibrin gel concentration is $30 \mathrm{mg} / \mathrm{mL}$ unless stated otherwise. (Adapted with permission from Price, G. M. et al. 2010. Biomaterials, 31, 6182-9; Wong, K. H. K. et al. 2013. J Biomed Mater Res A, 101, 2181-90.) 
balance between outward and inward radial stress at the vessel wall determines whether a vessel is stable. It also suggests that the adhesion stress $\sigma_{a d h}$ lies in the same range, which is consistent with measurements of the stress required to detach cultured cells from their substrata (Gallant et al. 2005).

Altogether, these data imply that the use of high-density, low-porosity scaffoldsfavored by many investigators for the ease of handling and fabrication-may require a well-designed drainage system to maintain vascular stability and perfusion. Fibrin gels with concentrations above $\sim 10 \mathrm{mg} / \mathrm{mL}$ ( $K$ below $\sim 10^{-9} \mathrm{~cm}^{4} / \mathrm{dyn} \cdot \mathrm{s}$ ) show considerable delamination in the absence of drainage, and we point out that other polymer matrices such as alginate and polyethylene glycol are even more hydraulically resistive by orders of magnitude (Comper and Zamparo 1989, Zimmermann et al. 2007).

\subsubsection{Prediction: Strong Endothelial Barrier Function Promotes Vascular Stability}

A third prediction of the mechanical model is that signals that promote strong endothelial barrier function will also promote stability. At first glance, the link between barrier function [typically quantified in terms of solute permeability coefficients (Michel and Curry 1999)] and physical stresses may not be obvious. The endothelial barrier, however, acts as a pathway for transport of both solutes and water; it is almost always the case that a barrier that leaks solutes will be hydraulically leaky as well (Rippe and Haraldsson 1994). When the hydraulic resistance of the vessel wall is low, the transmural pressure will also be low, all other parameters being equal. Thus, it is necessary for the vessel barrier to be strong if a large stabilizing transmural pressure is to be obtained.

In nearly all the vascular perfusion conditions that we have studied, stable vessels result only when the vessel wall restricts the movement of macromolecules of the size of serum albumin $(\sim 67 \mathrm{kDa})$. These barrier-strengthening conditions include addition of the antiinflammatory second messenger cyclic adenosine monophosphate (AMP) (Figure 6.5a) (Wong et al. 2010), addition of polymers such as dextran and hydroxyethyl starch (Leung et al. 2012), and elevation of vascular flow rate (Figure 6.5b) (Price et al. 2010). In the absence of such signals, the endothelium becomes leaky; in particular, it displays "focal" leaks that are localized to microscale regions on the vessel wall. When enough of these leaks open simultaneously, transmural pressure can decay on the timescale of minutes, as the vascular and scaffold pressures equilibrate (Wong et al. 2010). We have observed that barrierstrengthening conditions invariably result in vessels with strong junctional expression of vascular endothelial cadherin (VE-cadherin), which establishes a structural basis for the decreased permeability (Figure 6.5b) (Price et al. 2010).

Given the disparate nature of the stabilizing conditions described in this section, it is important to consider whether they promote stability through other mechanisms, besides strengthening the endothelial barrier. For instance, cyclic AMP is known to reduce endothelial cell contractility (Morel et al. 1989), and we did find considerable cell relaxation at the highest concentrations tested (Wong et al. 2010). Reduction in contractility, however, was not able to explain the stabilizing effect of cyclic AMP at lower concentrations. Also, polymers such as dextran are known to increase the colloid osmotic pressure of solutions; so, one might expect that this increase may itself exert a stabilizing effect. We think that an effect due to osmotic pressure is unlikely, because the scaffold is already saturated with the polymers by perfusion before a confluent endothelium forms, thereby eliminating a transvascular osmotic pressure difference. In fact, these polymers induce the elevation of VE-cadherin junctional localization even in endothelial cells that are grown on plastic substrates, a culture condition in which osmotic pressure differences should be negligible 
(a)

With fluorescent BSA
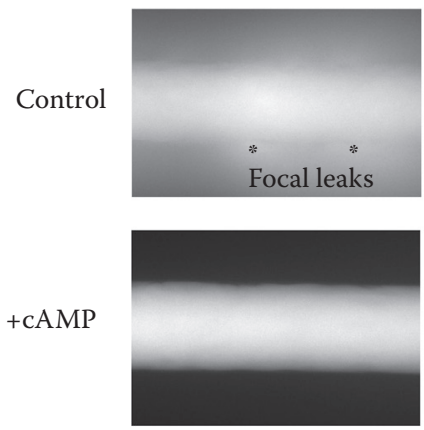

(b)

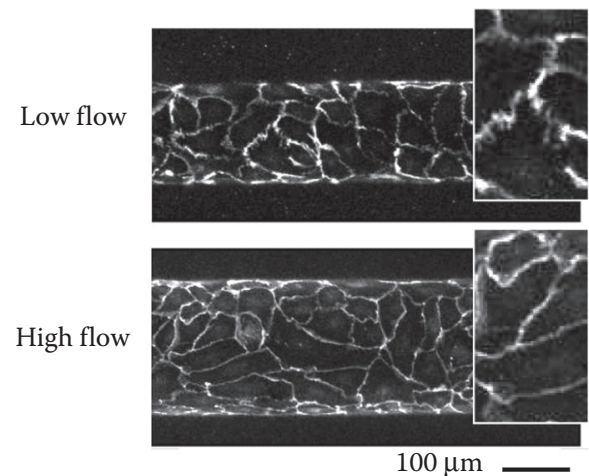

Phase-contrast

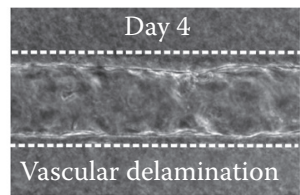

Transmural pressure maintained

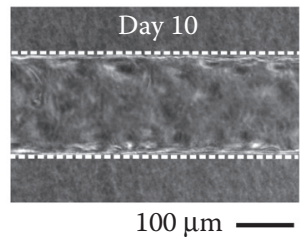

With fluorescent BSA
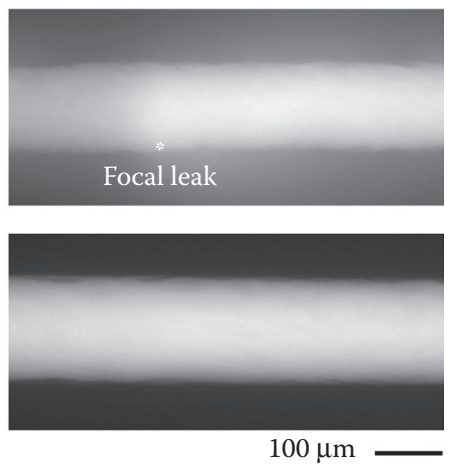

FIGURE 6.5

Conditions that prevent vascular leakage yield stable vessels. (a) Cyclic AMP, an anti-inflammatory second messenger, strengthens barrier function and maintains vessel stability. Barrier function was assayed on day 3 postseeding by taking fluorescence images of vessels perfused with Alexa Fluor 594-labeled bovine serum albumin (BSA). (b) High flow_-generated with a high-pressure gradient-enhances VE-cadherin localization at cell-cell junctions and reduces leakage in engineered vessels. Vessels under high flow are typically expanded (indicative of a high transmural pressure) and are stable. (Adapted with permission from Price, G. M. et al. 2010. Biomaterials, 31, 6182-9; Wong, K. H. K., Truslow, J. G. and Tien, J. 2010. Biomaterials, 31, 4706-14.)

(Leung et al. 2012). Finally, elevation of the flow rate can lead to simultaneous increases in shear stress and lumenal pressure. We have found that it is the increase in shear stress that leads to a decrease in endothelial permeability. The increase in lumenal pressure itself can directly increase transmural pressure. Thus, an increase in flow rate appears to stabilize in two complementary ways, by a direct change in transmural pressure and by maintenance of that pressure, which is only possible when the barrier function is strong (Price et al. 2010).

\subsubsection{Prediction: Scaffold Stiffening Promotes Vascular Stability Independently of Inhibition of Gel Contraction}

Finally, the mechanical model predicts that stiffening of scaffolds will stabilize vessels. The physical basis of this prediction lies in the nature of the delamination process. Although so far we have expressed the analysis as a phenomenological balance of stresses in Equation 6.3, an equivalent analysis views delamination via a balance of energies 
(Figure 6.3b) (Janssen et al. 2006). To detach endothelium from the scaffold, energy must be supplied to break the adhesive bonds between endothelial surface integrins and the underlying matrix proteins (i.e., $\delta E_{a d h}<0$ ). This energy can be supplied from the elastic recoil of the scaffold when the endothelium detaches (i.e., $\delta E_{\text {elastic }}>0$ ), as well as from the release of contractile energy as the surface area of endothelium decreases (i.e., $\delta E_{E C}>0$ ). In this formulation, the condition for stability becomes

$$
\delta E_{\text {elastic }}+\delta E_{a d h}+\delta E_{E C}<0
$$

When this inequality is satisfied, the energy released upon delamination is insufficient to overcome adhesion, and the vessel wall will be stable.

In principle, the elastic energy stored in the scaffold is inversely proportional to the scaffold stiffness for a given imposed stress (Beer et al. 2006). Thus, stiffening a scaffold reduces $\delta E_{\text {elastic, }}$ which makes delamination less energetically favorable. Studies of endothelial mechanobiology imply that scaffold stiffening can also lead to increases in total adhesion energy (which would promote stability) (Choquet et al. 1997) and contractility (which would promote delamination) (Huynh et al. 2011); we expect these secondary effects to largely offset.

In a recent work, we have analyzed how scaffold stiffening by the small-molecularweight cross-linker genipin affects vascular stability, and found that stiff scaffolds (moduli roughly fivefold greater than in native gels) promote stability as predicted (Chan et al. in press). Surprisingly, vessels in stiff scaffolds could withstand conditions that would normally lead to quick delamination, such as nearly zero-transmural pressure. To determine whether the energy-based mechanism described above is plausible, we have used computational modeling to obtain the elastic energy released upon endothelial delamination when transmural pressure is zero. In a scaffold of $400 \mathrm{~Pa}$ shear modulus (comparable to an $\sim 8 \mathrm{mg} / \mathrm{mL}$ native collagen gel), delamination of a $400 \mu \mathrm{m}^{2}$ patch of endothelium results in a release of $1.7 \times 10^{-6} \mathrm{erg}$ of energy, nearly all of which results from elastic recoil of the scaffold; typical adhesion strengths under slow peeling of $\sim 0.15 \mathrm{dyn} / \mathrm{cm}$ (Engler et al. 2004) yield an adhesion energy of $\sim 6 \times 10^{-7} \mathrm{erg}$, and we find that inequality (4) is not satisfied (i.e., delamination will occur). When the scaffold is stiff (shear modulus of $2 \mathrm{kPa}$ ), the elastic energy released upon delamination greatly decreases to $3.3 \times 10^{-7} \mathrm{erg}$, and now, inequality (4) is satisfied (i.e., delamination is inhibited). Thus, scaffold stiffness-induced stability is both experimentally observed and computationally realistic.

\subsection{Summary and Future Directions}

The discussion above summarizes the evidence for a physical mechanism of vascular stability in microfluidic scaffolds. Although other, nonphysical mechanisms (e.g., perivascular proteolysis of the scaffold) could also play a role in maintaining the endothelium, the success of a purely physical theory in explaining the effects of a wide range of signals on stability suggest that these alternative mechanisms are likely to be minor. Further clarification of the proposed mechanical stabilizing signals will benefit from direct, noninvasive measurements of transmural pressure, adhesion energy or stress, and cell contractility without relying on inference from computational modeling. Such in situ measurements 
are challenging, but may become possible with the development of fluorescent reporter proteins whose optical properties are altered by local mechanical strain (Klotzsch et al. 2009, Grashoff et al. 2010).

We have presented these ideas in the context of vascularizing microfluidic collagen and fibrin scaffolds. Might similar ideas hold in other systems of vascularization? It seems likely that vascularization of other microfluidic materials, such as synthetic polymer and alginate gels, should follow the same physical principles as those that govern vascularization of ECM-based scaffolds. The relative magnitudes of the relevant stresses or energies will depend on the particular chemistry of the material (e.g., $\sigma_{a d h}$ in synthetic polymer gels may be close to zero without coupling of adhesive peptides), and thus, the minimum transmural pressure required for stability may vary with scaffold chemistry. Since the density of cell adhesion ligands can be tailored on synthetic polymers, this class of scaffolds may be a particularly good model system for testing the effect of cell adhesion strength on vascular stability. In any case, we believe that the mechanical model of vascular stability will hold in all microfluidic scaffolds, including those that are formed by decellularization of native tissues (Badylak et al. 2011).

It is less clear to what extent this model can explain the signals that affect vascular stability in growth factor-induced angiogenesis, which is, by far, the most popular approach in vascularization. Several differences exist between the vascularization process described here and angiogenesis, most obviously in terms of scale (50- to $100-\mu \mathrm{m}$-diameter tubes vs. 5 - to $10-\mu \mathrm{m}$-diameter capillaries). In angiogenesis, the endothelial cells must create channels where none existed, and vascular regression can occur before and after initiation of perfusion through nascent sprouts (Black et al. 1998, Darland and D'Amore 2001, Shepherd et al. 2004). We suspect that the mechanical stress profile that surrounds a perfused capillary that arises by angiogenesis will depend on whether capillary sprouts grow primarily by expansion (i.e., by pushing against the surrounding matrix) or by migration into a just-formed channel. Moreover, vascular growth factors tend to activate endothelial cells and can induce hyperpermeability that may make transmural pressure less relevant as a stabilizing factor. Physical signals, such as shear stress, can stabilize capillaries through biochemical mechanisms, such as inhibition of apoptosis (Dimmeler et al. 1996). It remains to be determined whether physical signals play a major or minor mechanical role in stabilizing vessels that arise from angiogenesis.

Our results suggest that the ideal scaffold for microfluidic vascularization is one that is stiff and of intermediate pore size. The stiffer the scaffold, the more stable the vessels that form in it. With large pore sizes, the vascular wall becomes poorly defined as cells migrate from the channels into the scaffold; with small pore sizes, the hydraulic resistance of the scaffold destabilizes vessels. If small pore sizes are unavoidable, then addition of drainage channels to directly lower scaffold pressures is indicated. When designing scaffolds to engineer a vascularized tissue, these ideal characteristics may provide useful rules to guide the design process.

\section{Acknowledgments}

We thank John Hutchinson, Dimitrije Stamenović, and Victor Varner for insightful discussions of thin-film fracture mechanics. This work was supported by the National Heart, Lung, and Blood Institute through award HL092335. 


\section{References}

Alcaraz, J., Nelson, C. M., and Bissell, M. J. 2004. Biomechanical approaches for studying integration of tissue structure and function in mammary epithelia. J Mammary Gland Biol Neoplasia, 9, 361-74.

Asahara, T., Murohara, T., Sullivan, A., Silver, M., van der Zee, R., Li, T., Witzenbichler, B., Schatteman, G., and Isner, J. M. 1997. Isolation of putative progenitor endothelial cells for angiogenesis. Science, 275, 964-7.

Au, P., Tam, J., Fukumura, D., and Jain, R. K. 2008. Bone marrow-derived mesenchymal stem cells facilitate engineering of long-lasting functional vasculature. Blood, 111, 4551-8.

Badylak, S. F., Taylor, D., and Uygun, K. 2011. Whole-organ tissue engineering: Decellularization and recellularization of three-dimensional matrix scaffolds. Annu Rev Biomed Eng, 13, 27-53.

Barber, J. R. 2010. Elasticity. Dordrecht; New York: Springer.

Beer, F. P., Johnston, E. R., and DeWolf, J. T. 2006. Mechanics of Materials. Boston: McGraw-Hill.

Black, A. F., Berthod, F., L'Heureux, N., Germain, L., and Auger, F. A. 1998. In vitro reconstruction of a human capillary-like network in a tissue-engineered skin equivalent. FASEB J, 12, 1331-40.

Bouhadir, K. H. and Mooney, D. J. 2001. Promoting angiogenesis in engineered tissues. J Drug Target, 9, 397-406.

Chan, K. L. S., Khankhel, A. H., Thompson, R. L., Coisman, B. J., Wong, K. H. K., Truslow, J. G., and Tien, J. Crosslinking of collagen scaffolds promotes blood and lymphatic vascular stability. $J$ Biomed Mater Res A, in press.

Chen, C. S., Tan, J., and Tien, J. 2004. Mechanotransduction at cell-matrix and cell-cell contacts. Annu Rev Biomed Eng, 6, 275-302.

Chiu, L. L., Montgomery, M., Liang, Y., Liu, H., and Radisic, M. 2012. Perfusable branching microvessel bed for vascularization of engineered tissues. Proc Natl Acad Sci USA, 109, E3414-23.

Choi, N. W., Cabodi, M., Held, B., Gleghorn, J. P., Bonassar, L. J., and Stroock, A. D. 2007. Microfluidic scaffolds for tissue engineering. Nat Mater, 6, 908-15.

Choquet, D., Felsenfeld, D. P., and Sheetz, M. P. 1997. Extracellular matrix rigidity causes strengthening of integrin-cytoskeleton linkages. Cell, 88, 39-48.

Chrobak, K. M., Potter, D. R., and Tien, J. 2006. Formation of perfused, functional microvascular tubes in vitro. Microvasc Res, 71, 185-96.

Comper, W. D. and Zamparo, O. 1989. Hydraulic conductivity of polymer matrices. Biophys Chem, $34,127-35$.

Darland, D. C. and D'Amore, P. A. 2001. TGF beta is required for the formation of capillary-like structures in three-dimensional cocultures of 10T1/2 and endothelial cells. Angiogenesis, 4, 11-20.

Dimmeler, S., Haendeler, J., Rippmann, V., Nehls, M., and Zeiher, A. M. 1996. Shear stress inhibits apoptosis of human endothelial cells. FEBS Lett, 399, 71-4.

Discher, D. E., Janmey, P., and Wang, Y. L. 2005. Tissue cells feel and respond to the stiffness of their substrate. Science, 310, 1139-43.

Engler, A. J., Griffin, M. A., Sen, S., Bonnemann, C. G., Sweeney, H. L., and Discher, D. E. 2004. Myotubes differentiate optimally on substrates with tissue-like stiffness: Pathological implications for soft or stiff microenvironments. J Cell Biol, 166, 877-87.

Gallant, N. D., Michael, K. E., and Garcia, A. J. 2005. Cell adhesion strengthening: Contributions of adhesive area, integrin binding, and focal adhesion assembly. Mol Biol Cell, 16, 4329-40.

Gjorevski, N. and Nelson, C. M. 2010. The mechanics of development: Models and methods for tissue morphogenesis. Birth Defects Res C Embryo Today, 90, 193-202.

Golden, A. P. and Tien, J. 2007. Fabrication of microfluidic hydrogels using molded gelatin as a sacrificial element. Lab Chip, 7, 720-5.

Grashoff, C., Hoffman, B. D., Brenner, M. D., Zhou, R., Parsons, M., Yang, M. T., McLean, M. A. et al. 2010. Measuring mechanical tension across vinculin reveals regulation of focal adhesion dynamics. Nature, 466, 263-6. 
Huynh, J., Nishimura, N., Rana, K., Peloquin, J. M., Califano, J. P., Montague, C. R., King, M. R., Schaffer, C. B., and Reinhart-King, C. A. 2011. Age-related intimal stiffening enhances endothelial permeability and leukocyte transmigration. Sci Transl Med, 3, 112ra122.

Janssen, M., Zuidema, J., and Wanhill, R. J. H. 2006. Fracture Mechanics: Fundamentals and Applications. Delft: VSSD.

Kaully, T., Kaufman-Francis, K., Lesman, A., and Levenberg, S. 2009. Vascularization-The conduit to viable engineered tissues. Tissue Eng Part B Rev, 15, 159-69.

Klotzsch, E., Smith, M. L., Kubow, K. E., Muntwyler, S., Little, W. C., Beyeler, F., Gourdon, D., Nelson, B. J., and Vogel, V. 2009. Fibronectin forms the most extensible biological fibers displaying switchable force-exposed cryptic binding sites. Proc Natl Acad Sci USA, 106, 18267-72.

Koike, N., Fukumura, D., Gralla, O., Au, P., Schechner, J. S., and Jain, R. K. 2004. Tissue engineering: Creation of long-lasting blood vessels. Nature, 428, 138-9.

Kolodney, M. S. and Wysolmerski, R. B. 1992. Isometric contraction by fibroblasts and endothelial cells in tissue culture: A quantitative study. J Cell Biol, 117, 73-82.

Lee, J. and Schmid-Schönbein, G. W. 1995. Biomechanics of skeletal muscle capillaries: Hemodynamic resistance, endothelial distensibility, and pseudopod formation. Ann Biomed Eng, 23, 226-46.

Leung, A. D., Wong, K. H. K., and Tien, J. 2012. Plasma expanders stabilize human microvessels in microfluidic scaffolds. J Biomed Mater Res A, 100, 1815-22.

Ling, Y., Rubin, J., Deng, Y., Huang, C., Demirci, U., Karp, J. M., and Khademhosseini, A. 2007. A cellladen microfluidic hydrogel. Lab Chip, 7, 756-62.

Lovett, M., Lee, K., Edwards, A., and Kaplan, D. L. 2009. Vascularization strategies for tissue engineering. Tissue Eng Part B Rev, 15, 353-70.

Michel, C. C. and Curry, F. E. 1999. Microvascular permeability. Physiol Rev, 79, 703-61.

Miller, J. S., Stevens, K. R., Yang, M. T., Baker, B. M., Nguyen, D. H., Cohen, D. M., Toro, E. et al. 2012. Rapid casting of patterned vascular networks for perfusable engineered three-dimensional tissues. Nat Mater, 11, 768-74.

Morel, N. M. L., Dodge, A. B., Patton, W. F., Herman, I. M., Hechtman, H. B., and Shepro, D. 1989. Pulmonary microvascular endothelial cell contractility on silicone rubber substrate. J Cell Physiol, 141, 653-9.

Nichol, J. W., Koshy, S. T., Bae, H., Hwang, C. M., Yamanlar, S., and Khademhosseini, A. 2010. Cellladen microengineered gelatin methacrylate hydrogels. Biomaterials, 31, 5536-44.

Price, G. M., Chu, K. K., Truslow, J. G., Tang-Schomer, M. D., Golden, A. P., Mertz, J., and Tien, J. 2008. Bonding of macromolecular hydrogels using perturbants. J Am Chem Soc, 130, 6664-5.

Price, G. M. and Tien, J. 2009. Subtractive methods for forming microfluidic gels of extracellular matrix proteins. In: Bhatia, S. N., and Nahmias, Y., (eds.) Microdevices in Biology and Engineering. Boston, MA: Artech House.

Price, G. M. and Tien, J. 2011. Methods for forming human microvascular tubes in vitro and measuring their macromolecular permeability. Methods Mol Biol, 671, 281-93.

Price, G. M., Wong, K. H. K., Truslow, J. G., Leung, A. D., Acharya, C., and Tien, J. 2010. Effect of mechanical factors on the function of engineered human blood microvessels in microfluidic collagen gels. Biomaterials, 31, 6182-9.

Rippe, B. and Haraldsson, B. 1994. Transport of macromolecules across microvascular walls: The two-pore theory. Physiol Rev, 74, 163-219.

Schmid-Schönbein, G. W. 1990. Microlymphatics and lymph flow. Physiol Rev, 70, 987-1028.

Shepherd, B. R., Chen, H. Y., Smith, C. M., Gruionu, G., Williams, S. K., and Hoying, J. B. 2004. Rapid perfusion and network remodeling in a microvascular construct after implantation. Arterioscler Thromb Vasc Biol, 24, 898-904.

Tien, J., Wong, K. H. K., and Truslow, J. G. 2013. Vascularization of microfluidic hydrogels. In: Bettinger, C. J., Borenstein, J. T., and Tao, S. L., (eds.) Microfluidic Cell Culture Systems, 2nd edition. pp. 205-221. Oxford, U.K: Elsevier.

Tremblay, P.-L., Hudon, V., Berthod, F., Germain, L., and Auger, F. A. 2005. Inosculation of tissueengineered capillaries with the host's vasculature in a reconstructed skin transplanted on mice. Am J Transplant, 5, 1002-10. 
Truslow, J. G., Price, G. M., and Tien, J. 2009. Computational design of drainage systems for vascularized scaffolds. Biomaterials, 30, 4435-43.

Vailhé, B., Vittet, D., and Feige, J. J. 2001. In vitro models of vasculogenesis and angiogenesis. Lab Invest, 81, 439-52.

Wong, K. H. K., Chan, J. M., Kamm, R. D., and Tien, J. 2012. Microfluidic models of vascular functions. Annu Rev Biomed Eng, 14, 205-30.

Wong, K. H. K., Truslow, J. G., Khankhel, A. H., Chan, K. L., and Tien, J. 2013. Artificial lymphatic drainage systems for vascularized microfluidic scaffolds. J Biomed Mater Res A, 101, 2181-90.

Wong, K. H. K., Truslow, J. G., and Tien, J. 2010. The role of cyclic AMP in normalizing the function of engineered human blood microvessels in microfluidic collagen gels. Biomaterials, 31, 4706-14.

Yeon, J. H., Ryu, H. R., Chung, M., Hu, Q. P., and Jeon, N. L. 2012. In vitro formation and characterization of a perfusable three-dimensional tubular capillary network in microfluidic devices. Lab Chip, 12, 2815-22.

Zheng, Y., Chen, J., Craven, M., Choi, N. W., Totorica, S., Diaz-Santana, A., Kermani, P. et al. 2012. In vitro microvessels for the study of angiogenesis and thrombosis. Proc Natl Acad Sci USA, 109, 9342-7.

Zimmermann, H., Wahlisch, F., Baier, C., Westhoff, M., Reuss, R., Zimmermann, D., Behringer, M. et al. 2007. Physical and biological properties of barium cross-linked alginate membranes. Biomaterials, $28,1327-45$. 